\title{
Fast Retrieval of Similar Configurations
}

\author{
Dimitris PAPADIAS, Marios MANTZOUROGIANNIS, and Ishfaq AHMAD
}

\begin{abstract}
Configuration similarity is a special form of contentbased image retrieval that considers relative object locations. It can be used as a standalone method, or to complement retrieval based on visual or semantic features. The corresponding queries ask for sets of objects that satisfy some spatio-temporal constraints, e.g., "find all triplets of objects $\left(v_{1}, v_{2}, v_{3}\right)$ such that $v_{1}$ is northeast of $v_{2}$ which is inside $v_{3}$ ". Exhaustive processing (i.e., retrieval of the best solutions) of configuration similarity queries, in general, has exponential complexity and fast search for suboptimal solutions is the only way to deal with the vast amounts of multimedia information in several real-time applications. In this paper we first discuss the utilization of non-systematic search heuristics, based on genetic algorithms, simulated annealing and hill climbing approaches. An extensive experimentation with real and synthetic datasets reveals that hill climbing techniques are the best for the current problem; therefore, as a subsequent step we study the search space, and develop improved variations of hill climbing that take advantage of the special structure of the problem to enhance speed. The proposed heuristic methods significantly outperform systematic search when there is only limited time for query processing.
\end{abstract}

Index Terms-- Content-based retrieval, spatial similarity, local search algorithms.

\section{INTRODUCTION}

$\mathrm{T}$ he large availability of visual content in emerging multimedia applications and the WWW has triggered significant advances in content-based retrieval mechanisms. Such mechanisms, sometimes in conjunction with traditional information retrieval techniques for text, allow users to access a variety of information sources. A special form of contentbased retrieval is configuration similarity, also known as spatial, structural, or arrangement similarity. The corresponding queries describe some prototype configuration and the goal is to retrieve all images containing arrangements of objects matching the input exactly or approximately. As an example consider that the user is looking for all images (video frames, html pages, VLSI circuits) containing arrangements similar to those of Fig. 1(a). Such a query could be expressed by one of the existing pictorial languages that permit configuration similarity retrieval, e.g., VisualSeek [34], Query by Sketch [8], PQBE [29], Safe [35], or extended SQL commands, e.g., Select $v_{0}, v_{1}, v_{2}, v_{3}$, From ImageDB, Where

Manuscript received April ?, 2000. This work was supported by grants HKUST 6070/00E and HKUST 6081/01E from Hong Kong RGC. Parts of the paper appear in [25][28].

Department of Computer Science, Hong Kong University of Science and Technology, Clearwater Bay, Hong Kong (telephone: +852-23586971, e-mail: \{dimitris, mantzour, iahmad\} cs.ust.hk).
$\mathrm{NE}\left(v_{0}, v_{1}\right), \mathrm{NW}\left(v_{0}, v_{2}\right), \mathrm{N}\left(v_{0}, v_{3}\right), \ldots$ (NE means northeast, NW northwest, and so on).

Formally, a configuration similarity query can be described by: (i) a set of $n$ variables, $v_{0}, v_{1}, \ldots, v_{n-1}$ that appear in the query; (ii) for each variable $v_{i}$, a finite domain $D_{i}=\left\{u_{0}, \ldots, u_{N i-}\right.$ $\left.{ }_{1}\right\}$ of $N_{i}$ values; (iii) for each pair of variables $\left(v_{i}, v_{j}\right)$, a constraint $C_{i j}$ which can be a simple spatio-temporal relation or a disjunction of relations. The example query contains four variables $\left(v_{0}, \ldots, v_{3}\right)$, one for every drawn object. The domain of each variable consists of the objects in the image(s) to be searched for the particular configuration. The input constraints restrict the possible assignments of variables to subsets of the domains. In addition to binary spatio-temporal relations, some query languages allow the user to specify unary constraints in the form of object properties at the feature ( $v_{0}$ is a red square) or the semantic level ( $v_{0}$ is a building). In this case, appropriate retrieval algorithms (e.g., for color matching) must be integrated with the ones for configuration similarity.

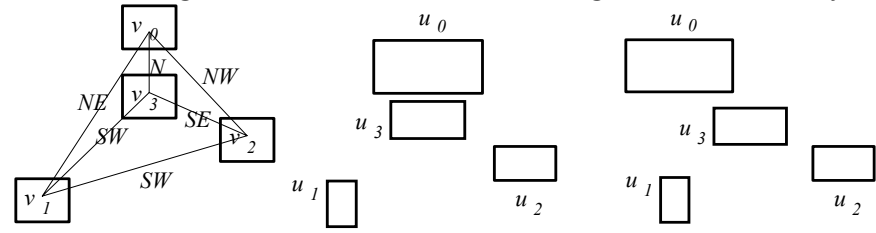
(a) query
(b) a perfect match
(c) approximate answer

As in most forms of information retrieval, a scoring mechanism should be employed for inexact matches. Depending on the types of constraints allowed in the expression of queries, several types of similarity measures have been proposed. [22] uses Allen's [1] relations in multidimensional space and conceptual neighborhoods [10]. The idea is extended in [28] with the incorporation of binary string encoding to automate similarity calculations. Conceptual neighborhoods for topological relations (e.g., inside, overlap) are also applied in [8]. [15] uses angular directions (e.g., northeast is defined as an angle of 45 degrees) and fuzzy similarity measures. A related approach, which also includes distances between object centroids, is followed in [27].

Irrespective of the relations employed and the similarity measures used, the goal of query processing is to find instantiations of variables to image objects so that the input constraints are satisfied to a maximum degree. The inconsistency degree $d_{i j}$ of a binary instantiation $\left\{v_{i} \leftarrow u_{k}, v_{j} \leftarrow\right.$ $\left.u_{l}\right\}$ is defined as the dissimilarity between the relation $R\left(u_{k}, u_{l}\right)$ (between objects $u_{k}$ and $u_{l}$ in the image to be searched) and the 
constraint $C_{i j}$ (between $v_{i}$ and $v_{j}$ in the query). Given the inconsistency degrees of binary constraints, the inconsistency degree $d(S)$ of a complete solution $S=\left\{v_{0} \leftarrow u_{p}, . ., v_{i} \leftarrow u_{k}, . ., v_{j}\right.$ $\leftarrow u_{l}, . ., v_{n-1} \leftarrow u_{r}$ \} can be defined as:

$$
d(S)=\sum_{\forall i, j, i \neq j \text { and } 0 \leq i, j<n} d_{i j}\left(C_{i j}, R\left(u_{k}, u_{l}\right)\right) \text { where }\left\{v_{i} \leftarrow u_{k}, v_{j} \leftarrow u_{l}\right\}
$$

Fig. 1(b) and (c) illustrate two solutions for the example query where $v_{i} \leftarrow u_{i}, 0 \leq i<4$. The first solution corresponds to a perfect match, while the second is inexact since some binary constraints (e.g., between $v_{0}$ and $v_{l}$ ) are not totally satisfied. If $N$ is the image cardinality, the total number of possible solutions that have to be considered in each image is equal to the number of $n$-permutations of the $N$ objects: $N ! /(N-n)$ !. Due to the high cost of query processing, it is not always possible to search all database images within a reasonable amount of time. In some cases, systematic search for the best solutions even in a single large image may take hours to complete [27].

An alternative approach is to compromise quality in order to achieve speed; in other words, we could assign a certain amount of processing to each image (possibly proportional to its size or importance) so that the whole database can be searched within the available time. In this paper we follow this approach and exploit non-systematic search heuristics that can quickly provide good, but not necessarily optimal, solutions. The contributions of this work can be summarized as follows: (a) we first apply three search methodologies, genetic algorithms, hill climbing and simulated annealing, to the problem of configuration similarity retrieval and identify the best one (hill climbing) using a variety of experimental settings; (b) we perform a study of the solution space and evaluate alternative search strategies for hill climbing and (c) based on our study, we develop improved algorithms that take advantage of the spatial structure of the problem to enhance performance.

The rest of the paper is organized as follows: Section 2 outlines previous processing approaches and discusses their advantages and shortcomings. Section 3 describes the application of genetic algorithms, hill climbing and simulated annealing, to configuration similarity retrieval. Section 4 compares systematic search with non-systematic search, using experiments with both synthetic and real datasets. Section 5, studies the problem space and proposes improved variations of hill climbing. Section 6 concludes the paper with a discussion.

\section{QUeRY PROCESSING TECHNIQUES}

The problem of configuration similarity retrieval is similar to scene matching, which has been extensively studied in computer vision and pattern recognition [32][33]. In the multimedia databases literature, several forms of processing configuration similarity, based on different assumptions and algorithms, have been proposed. The various approaches can be classified according to the size of database images for which they can be applied, the form of relations permitted, and the type of query variables. The form of relations, otherwise called relation scheme, can be static or dynamic. Static methods assume a predefined set of relations to be used by all users in all queries. Dynamic methods can be employed with any type of relations (assuming of course that the query language allows different sets of relations for different queries). Query variables can be fixed or unrestricted: a fixed variable can be instantiated to at most one object in each image, while an unrestricted one can range within the whole domain.

A class of methods, which can be grouped under a general category called pairwise matching, assumes that all query variables are fixed (e.g., find all images in which George is left of Mary). Thus, an image has at most one configuration matching the query which can be found in polynomial time as follows: (a) locate the query objects in the image (possibly using an index on object id), (b) for each object pair compute its similarity to the corresponding query constraint, and (c) calculate the total similarity of the configuration using the pairwise similarities. [15] follows this approach to answer configuration similarity queries involving angular directions including rotation invariants. [22] deals with projection directions and topology. Algorithms that combine pairwise matching with contextual similarity (i.e., based on object features) can be found in [37]. Assuming that image objects are stored using absolute coordinates, pairwise matching can be applied with dynamic relation schemes. Its disadvantage is its limited applicability due to the fixed nature of query variables.

[30] solves configuration queries for medical images (Xrays) that contain a constant number of labeled/expected objects (e.g., stomach, heart) and a small number of unlabeled ones (e.g., tumors). Every image is mapped onto a point in multi-dimensional space, where each dimension corresponds to a relation between a specific pair of objects; i.e., if $N$ is the number of image objects and $r$ the number of relations in the relation scheme, the number of dimensions is $O\left(r N^{2}\right)$. Queries, which are also X-ray images containing mostly labeled (i.e., fixed) variables, are processed by multidimensional nearest neighbor search using R-trees. In order to keep the number of dimensions stable, images with unlabelled objects are decomposed into combinations of images with fixed size. An enhanced version that reduces the number of dimensions is proposed in [31]. Performance could be further improved by employing more efficient high dimensional indexing methods, such as $M$ trees [6], the pyramid technique [2] etc. Nevertheless, the method (like all techniques based on high dimensional indexing and search) is applicable only for static relation schemes (otherwise it is not possible to pre-determine the dimensions) and databases with small images (fewer than 10 objects) of mainly labeled objects (otherwise, the number of dimensions and images explodes to unmanageable levels).

A number of methods are based on several variations of 2D strings, which encode the arrangement of objects on each dimension into sequential structures. $2 D B$ strings capture the object projections, while $2 D C$ and $2 D G$ strings decompose objects in entities with disjoint convex hulls, allowing the 
representation of more detailed spatial information at the expense of storage [3][19][20]. Every database image is indexed by a $2 \mathrm{D}$ string, and queries are also transformed to 2D strings while configuration similarity retrieval is performed by applying appropriate string matching algorithms [4]. If the query contains only fixed variables, the cost of processing each image is polynomial, while in the general case it is exponential since matching has to be performed for multiple instantiations of the variables to different image objects. Users are not allowed to define their own relation scheme, but are restricted to the relations captured by the 2D strings.

[27] deals with configuration similarity without any restriction on the type of variables or relations. Approximate retrieval is modeled and solved as a constraint satisfaction problem by applying branch and bound algorithms that stop searching once a partial solution cannot lead to a desired target. The method is applicable for images of $10^{2}-10^{3}$ objects and can be employed with variable relation schemes. In [26], the incorporation of spatial indexing (R-trees) enables retrieval from much larger images $\left(10^{4}-10^{5}\right.$ objects). Although this approach works well in most cases, systematic search algorithms do not have a predictable behavior depending on the problem size. Different query/image combinations, even with the same number of variables and image objects, may yield vast variances in cost depending on constrainedness [12]. For instance, the running time for the same query in two images of the same size may be orders of magnitude different. As a consequence, a large part of query processing may be devoted to a few images, while other images may not be searched at all within the available time.

\section{HeUristic SEARCH For CONFIGURATION SimiLARITY}

Consider a database with numerous, large images where users can ask any type of queries (i.e., with non-fixed variables) using variable relation schemes. The only approach that could be employed here is systematic search [26][27], which due to the worst-case exponential cost is not guaranteed to terminate within reasonable time. In order to deal with configuration similarity under limited time, we employ search heuristics based on genetic algorithms, hill climbing and simulated annealing, which are explained as follows.

\section{A. Genetic Algorithms}

Genetic algorithms [13] are based on the concepts of natural mutation and the survival of the fittest individuals. Given a well-defined search space, three different genetic operations, selection, crossover and mutation, are applied to transform an initial population of chromosomes to next generation with the objective to improve their quality. A chromosome is an encoded representation of a feasible solution (i.e., in our problem an assignment of each query variable to an image object). Before the search process starts, a set of $P$ chromosomes (called initial population) is initialized to form the first generation. Then the three genetic search operations are repeatedly applied in order to obtain a population (i.e., a new set of solutions) with better characteristics, on which the genetic algorithm performs the same actions and so on, until a stopping criterion is met.

Next we demonstrate a genetic configuration similarity algorithm (GCSA), by presenting the encoding mechanism and then the selection, crossover and mutation operators.

Encoding mechanism: Each chromosome/solution is simply an array $S$ of $n$ values, where $S[i]$ is the instantiation of variable $v_{i}$ in solution $S$. The quality of $S$ is measured by its fitness $f$ (i.e., its similarity). $F$ is the average fitness of a population of chromosomes.

Selection mechanism: This operation consists of two parts: evaluation of a chromosome and offspring allocation. Evaluation is performed by measuring the above defined fitness value; offspring generation is then done by allocating to each chromosome, a number of offspring proportional to its fitness. GCSA implements the stochastic remainder technique [36]: a solution is assigned offspring according to the integer part of the proportionate fitness $(f / F)$ value in a deterministic way and the fractional parts are put in a roulette wheel ${ }^{1}$ for determining the remaining offspring. Thus, we restrict randomness to the fractional parts only and assure that a good chromosome will not vanish.

Crossover mechanism is the driving force in the exploration part of a genetic algorithm. In the simplest approach, pairs of chromosomes are selected randomly from the population. For each pair a crossover point is defined randomly, and the chromosomes beyond it are mutually exchanged, with probability $\mu_{\mathrm{c}}$ (crossover rate), producing two new chromosomes. The two newly generated chromosomes are very likely to possess the good characteristics of their parents (building-block hypothesis [13]). In our case this corresponds to swapping of the assignments in two solutions after a selected point. One-point crossover is inefficient for our application domain, since the probability of a bit to be swapped increases as we move to the end of the string. Instead we selected a two-point crossover mechanism for GCSA: after the pairing of chromosomes, two crossover points are randomly selected and the portion of the chromosome in between them is swapped. The entire operation is performed with probability $\mu_{\mathrm{c}}$.

Mutation mechanism: Mutation aims at restoring lost genetic material and is performed in GCSA by simply changing a variable instantiation with a probability $\mu_{\mathrm{m}}$, called the mutation rate. Although mutation is not the primary search operation and sometimes is omitted, it may be very useful for exploitation, i.e., cases where, through selection and crossover, all the chromosomes have converged to a local optimum for some variable.

GCSA starts with an initial population of $P$ randomly generated chromosomes / solutions and terminates after the creation and evaluation of $G$ generations. If only one solution

\footnotetext{
${ }^{1}$ Roulette wheel selection allocates a sector of the wheel equal to $2 \pi f / F$ to every chromosome and then creates an offspring if a generated number in the range of 0 to $2 \pi$, falls inside the assigned sector of the chromosome.
} 
is needed, then the best chromosome among all generations is returned. The option of specifying a target fitness also exists (i.e., retrieve the best $K$ solutions where the similarity is greater than target), in which case only chromosomes that exceed the target are kept at each run of GCSA.

Several theoretical and empirical studies [14][36] have been carried out on the control parameters, $P, G, \mu_{\mathrm{c}}$ and $\mu_{\mathrm{m}}$. Most results suggest that the mutation and the crossover rate should be in the range of $0.001 \%-0.05 \%$ and $0.60-0.95$, respectively. We experimented with these values using the queries and datasets described in Section 4. The best results for most cases were achieved for $\mu_{\mathrm{m}}=0.05 \%$ and $\mu_{\mathrm{c}}=0.60$. We measured the average similarity of the best solutions (for all combinations of queries and datasets) found by GCSA for several values of $P$ in the range $50-300$ and $G$ in the range 500-100 (as $P$ increases, $G$ has to decrease in order to keep the execution time constant). Fig. 2 shows the fitness of the solution as a function of $P$ and $G$.

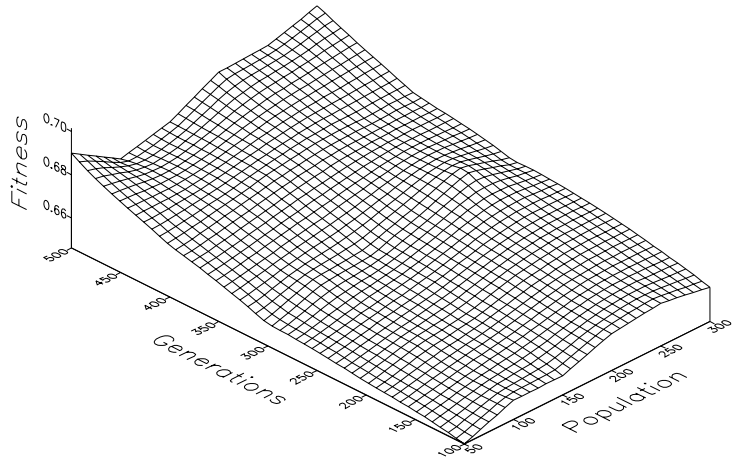

Fig. 2. Fitness (similarity) as a function of $P$ and $G$ in GCSA

The different values of $P$ do not affect fitness significantly; we chose $P=50$ because this value produces fair results for all cases and is small enough to allow a sufficient number of generations even for large problem instances. Combined with the relatively high value of $\mu_{\mathrm{c}}(0.60)$ GCSA was able to exploit a large portion of the solution space.

\section{B. Hill Climbing}

The problem space for configuration queries can be thought of as a graph, where each solution corresponds to a node associated with a similarity value. The goal is to find the nodes with the globally maximum similarity, i.e., the best solutions. Hill climbing algorithms operate on such a graph, performing random walks between the nodes based on a certain movement (transition) mechanism. This transition mechanism defines a neighborhood for each node $S$, which consists of all the nodes that can be reached from $S$ in one move. In our case, the neighbors of $S$ are all the solutions that can be derived from $S$ by changing the assignment of a single variable, i.e., a node has $n(N-1)$ neighbors, where $n$ is the number of query variables and $N$ the image cardinality (each variable can take $N-1$ values, excluding its current assignment). A move is called uphill, if it leads to a better solution, and downhill if the destination node has lower similarity.

Configuration similarity iterative improvement (CSII) starts with a randomly chosen initial solution (seed) and tries to find a better one by visiting random neighbors. If such a solution is found, it replaces the previous one. The process continues until a local maximum is reached. This iterative optimization is repeated a number of times, each time starting from a different seed. The user defines the stopping criterion by specifying the running time, or providing the target similarity of the solutions to be retrieved. As time approximates $\infty$, the probability that iterative improvement will find the global maximum approximates 1 [23]. However, given a finite amount of time, the algorithm terminates at a local maximum.

\section{Simulated Annealing}

Configuration similarity simulated annealing (CSSA), based on [5][18], also performs random walks, but in addition to uphill, it also accepts downhill moves with a certain probability. The intuition behind accepting downhill moves is led by the fact that some local maxima may be close to each other, separated by a small number of downhill moves. If only uphill moves were accepted (as in CSII) the algorithm would stop at the first local maximum visited, missing a subsequent (and possibly better) one. Fig. 3 illustrates CSSA for the case where the user requires solutions exceeding the similarity specified by target.

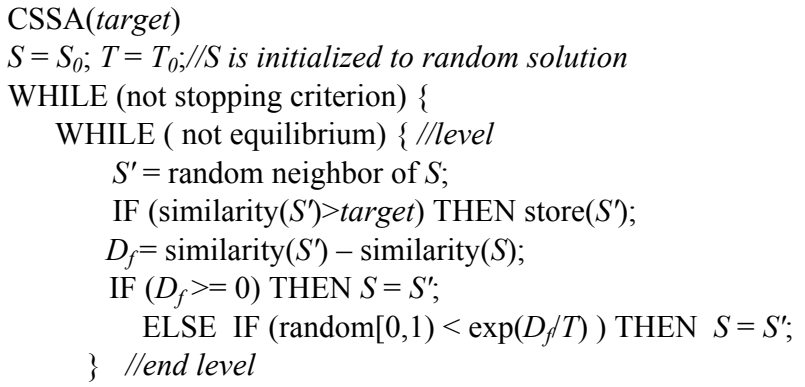

Fig. 3. The outline of CSSA

The inner for-loop is called level. Each level is executed with a fixed value of the parameter $T$. The starting value of $T$ is such that the probability $\exp \left(D_{f} / T\right)$ at the first levels approximates 1 , where $D_{f}$ denotes the difference between the similarity of the current solution $S$ and the new random neighbor $S^{\prime}$. After the execution of each level, $T$ is reduced according to some function, and the next level is performed using the new value of $T$. This means that the probability of accepting a downhill move is greater at the earlier levels and decreases in the subsequent ones. CSSA terminates when the value of $T$ is very close to zero and thus the probability of accepting downhill moves is almost 0 . Another way for the algorithm to stop is when a fixed criterion is reached; for example, when a solution with a given target similarity has been found.

As with GCSA, the quality of the output is strongly related to the choice parameter values. In order to define the initial value $T$ we adopt the method of [18]: a large value for $T_{0}$ is chosen and a number of transitions are performed. If the 
acceptance ratio $x$, defined as the number of accepted transitions divided by the number of proposed transitions, is less than a given value $x_{0}$ (in [18], $x_{0}=0.8$ ), $T_{0}$ is doubled. This procedure continues until the acceptance ratio exceeds $x_{0}$. Experimental evaluation suggests that $x_{0}=0.8$ and a $T_{0}$ equal to the similarity of the initial solution, is the best combination for the initial value of $T$. For decreasing the value of $T$, we apply the common (e.g., [17]) decrement rule: $T_{k+1}=a * T_{k}$, where $a=0.95$.

The length of the inner while-loop is determined by the equilibrium condition. For a given value of $T$, an equilibrium is reached if all the neighbors of a solution $S$, have the same similarity with $S$. This parameter is, in general, the most complicated to adjust because it is closely related to the specific problem. We experimented using several queries with various sizes, over multiple datasets. The following formula provides a suitable value for the number of iterations:

$$
\log (P(N, n))=\log \left(\frac{N !}{(N-n) !}\right)
$$

where $P(N, n)$ is the number of $n$-permutations of the $N$ objects (i.e., the number of possible solutions). (2) has been widely used in the constraint satisfaction literature as a measure of the problem size [12].

\section{EXPERIMENTAl Evaluation of SEARCH Heuristics}

In order to evaluate performance, we constructed five sets, each containing 25 queries using the relation scheme and similarity measures ${ }^{2}$ of [7]. The number of variables in each set was fixed to 3, 6, 9, 12 and 15. Query tightness varied from complete queries (where all pairs of variables are constrained) to very loose ones involving only a few nonrestrictive constraints. We used the three 2D datasets in Fig. 4; the first set contains randomly generated rectangles according to a uniform distribution, while the second set contains a VLSI circuit, and the third set contains road segments of Greece. Note that the density (sum of all rectangle areas divided by the workspace) and distribution of the objects significantly affects the performance of algorithms since it determines the quality of solutions. For instance, queries involving constraints such as overlap, inside, etc., are more easily satisfied in the second dataset due to its high density. Heuristic search is especially sensitive to the number of solutions [12]; if there exist only a few good solutions (e.g., for some restrictive large queries) it requires a significant amount of time to find them. The above datasets cover a wide range of cardinality values, data densities and distributions; thus they provide a good estimation for the performance of the algorithms on most problem instances.

As a benchmark for systematic search we used forward

\footnotetext{
${ }^{2}$ The algorithms can be employed with any type of spatial constraints. Due to the absence of a standard benchmark, we use the relation scheme in [7] since: (a) it is general, in the sense that it can express all types of common constraints (i.e., direction, topological and distance), (b) it permits the automatic calculation of similarity measures, and (c) it has been used for systematic search algorithms [26].
}

checking (FC) [16], because it is considered to be one of the most effective algorithms for general CSP problems, as well as for configuration similarity [26,27]. The current implementation of FC works in a branch and bound manner, i.e., a partial solution is abandoned if it cannot lead to similarity equal or higher than the best already found. In this way unsuccessful instantiations are rejected early and the search space is pruned effectively. We also compare performance with random sampling (RND), which chooses solutions randomly and keeps the best ones - for some optimization problems, random sampling outperforms other search heuristics due to its simplicity [11]. The experiments were run on a SUN UltraSparc2 $(200 \mathrm{MHz})$ with $256 \mathrm{MB}$ of RAM.

The first set of experiments measures the CPU time (in milliseconds) required to find one solution with similarity ${ }^{3}$ above a target of $0.7,0.75$ and 0.8 . Each execution was allowed 400 seconds to complete; after this period it was terminated. Fig. 4 illustrates the results for every query size/dataset combination (each row corresponds to one query size and each column to one dataset). CSII and CSSA clearly outperform the other algorithms for all cases, with CSII being the best option. Moreover, these algorithms were the only ones to successfully terminate for all combinations; FC and RND exceeded the time threshold in most large queries, and their results are omitted from most graphs. RND, in general, outperformed FC. This is because RND, due to its simple implementation, checks more instantiations per second than the other algorithms. GCSA, on the average, yields slightly better performance than RND, but in comparison to CSII and CSSA requires more time to reach a solution above the target similarity.

The performance of all algorithms degrades as the query size increases because large queries have, in general, few good solutions and a large part of the space has to be searched. The algorithms are most effective in the second dataset due to the presence of a high number of solutions, especially for small queries (note that RND always finds solutions for target similarity 0.7 ). Obviously, if the available processing time increases, FC will eventually outperform nonsystematic search - but for the current, rather long (400 seconds) processing limit, it is inefficient.

The next set of experiments measures the similarity of the best 50 solutions retrieved by the algorithms as a function of the execution time (50, 100, 150 and 200 seconds). Each diagram in Fig. 5 corresponds to a different query size and shows the similarity ranges of the 50 solutions averaged over the three datasets of Fig. 4. In other words, the lowest (highest) value represents the average of all lowest (highest) similarities for queries of the given size in any dataset.

\footnotetext{
${ }^{3}$ Similarity values range between 0 and 1 . Some queries (especially large ones) do not have perfect matches.
} 

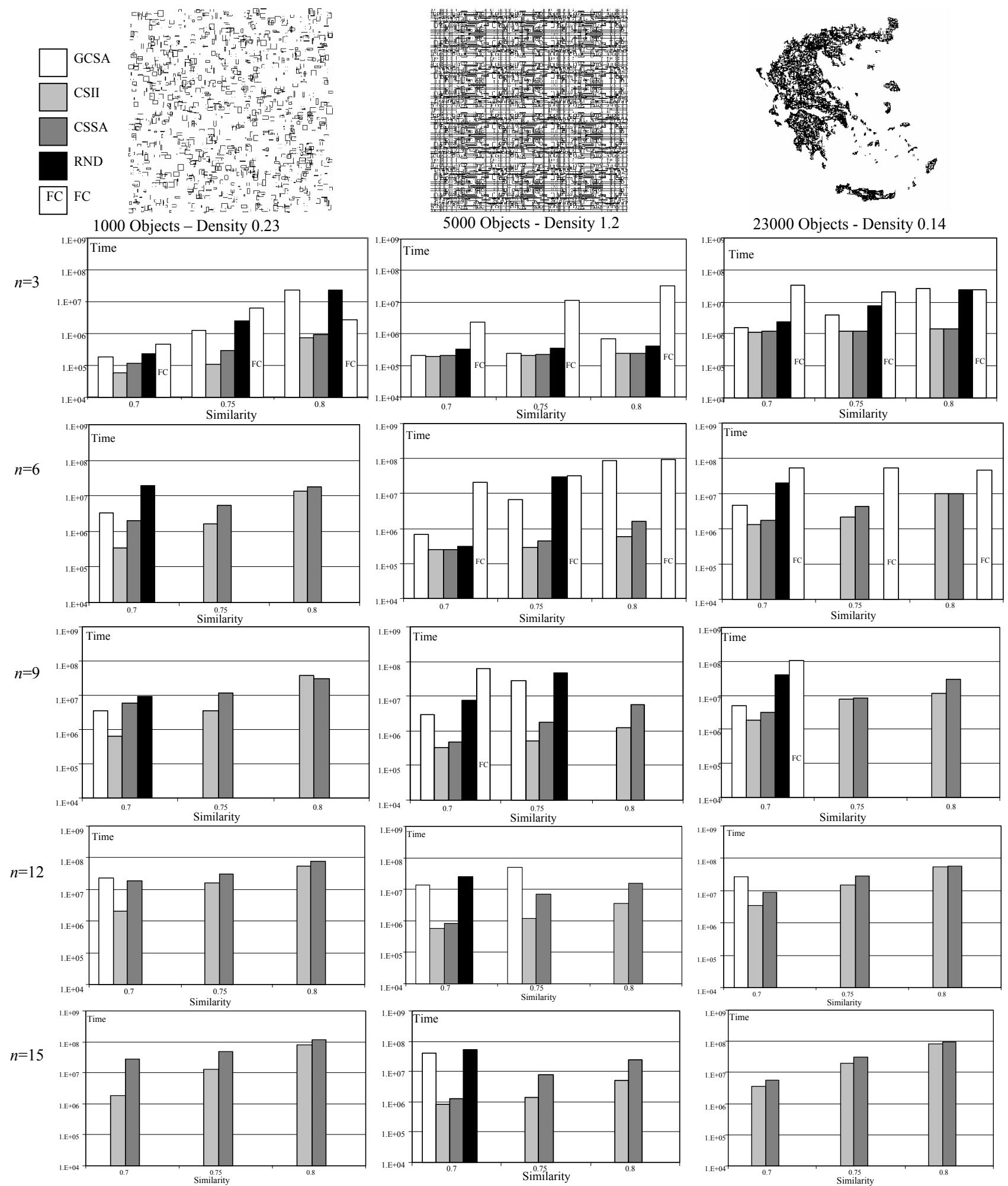

Fig 4. Time (msec) required to retrieve a solution with a given target similarity

As expected, CSII and CSSA again outperform the other algorithms. The greater range of similarity values for CSSA can be explained by the fact that it starts from a random solution, which tends to have low similarity and remains in this region, until the temperature is reduced significantly. CSII also starts from a solution with low similarity, but very soon reaches a region with high similarity because it accepts only better solutions. Therefore, it has a better performance for the current problem because it can reach very quickly a local maximum while SA spends the initial stages exploiting low similarity regions.

GCSA performs better than RND but the quality of retrieved solutions with respect to CSII and CSSA drops for large queries (where the number of good solutions is small). 

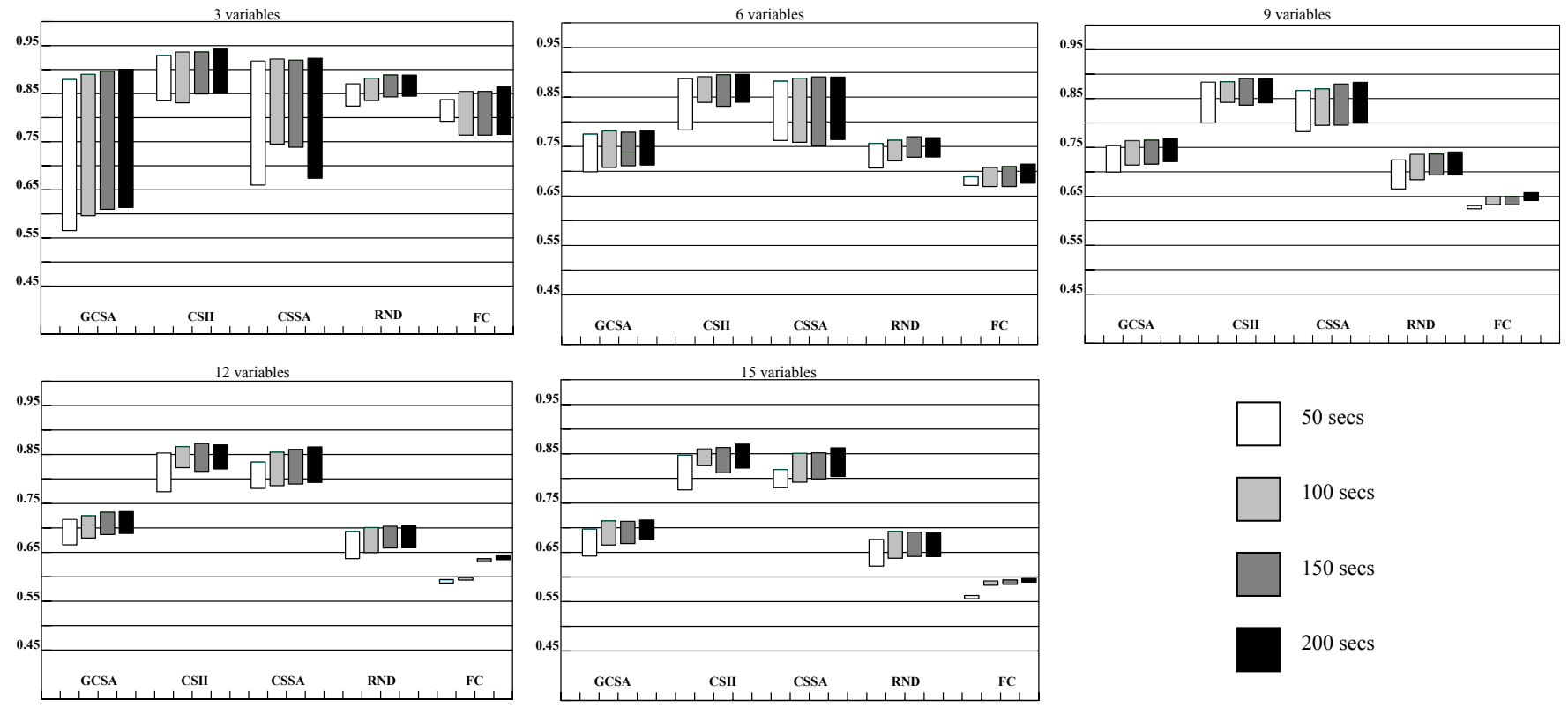

Fig. 5. Similarity range of 50 best solutions for pre-determined execution time

The wide range of similarities for queries with three variables can be explained by the fact that if a single instantiation changes (e.g., due to mutation), it significantly affects (up to $33 \%$ ) the fitness of the solution. FC is acceptable only for queries involving three variables where there is enough time to search a good part of the solution space. Its performance deteriorates significantly with the query size. It can be observed that that for large queries all solutions retrieved are in a narrow and low similarity range. This is because in restricted time periods, FC will find an area of the search space where some constraints are partially satisfied (while the rest totally violated) and retrieve all 50 solutions in this area. Most of these solutions tend to have the same instantiations for the partially satisfied constraints and differ only on the remaining variables.

In summary, non-systematic clearly outperforms systematic search when the processing time is limited with respect to the problem size. Among the heuristics tested, CSII yields the best performance. Furthermore, it does not require parameter tuning. This is a significant advantage because tuning of GCSA and CSSA is rather complicated. Moreover, the parameter values depend on the query and dataset characteristics (i.e., a set of values that yield optimal results for a query/image combination, may provide very poor results for another combination). Subsequently, we further improve the performance of hill climbing by proposing alternative search strategies.

\section{Improved Hill Climbing AlgorithmS}

CSII generates neighbors by selecting a random variable and changing its instantiation. An alternative approach, motivated by conflict minimization algorithms [21] is to select the "worst" variable. The inconsistency degree of a variable $v_{i}$ (currently instantiated to value $u_{k}$ ) in a solution $S$ is defined as the sum of inconsistency degrees of all binary instantiations involving $v_{i}$ :

$$
d\left(v_{i}, S\right)=\sum_{\forall j, i \neq j \text { and } 0 \leq j<n} d_{i j}\left(C_{i j}, R\left(u_{k}, u_{l}\right)\right) \text { where }\left\{v_{j} \leftarrow u_{l}\right\}
$$

Worst variable selection re-instantiates the variable with the highest inconsistency degree, so that the similarity of the specific solution may be increased significantly. If the worst variable cannot be improved, the second worst will be considered and so on. If one variable can be improved, the next step will consider again the new worst one; otherwise, if all variables are exhausted with no improvement, the current solution is considered to be a local maximum.

Once a variable is chosen for re-instantiation, CSII determines its new value by applying first-better value selection, i.e., by assigning values to the specific variable randomly, until a better instantiation is found. When the similarity of a solution is very low, first-better selection performs just a few attempts before it finds a better solution. As the quality increases, it becomes more difficult for the solution to be improved by random re-instantiations. If after $N$ unsuccessful assignments no better neighbor is found, the solution is considered a local maximum. Notice, however, that due to the random nature of search, better neighbors may be missed since some instantiations are tried multiple times, whereas others not at all. Another option is all-best value selection (sometimes called steepest ascent), which systematically tries all possible values in the domain of the variable to be re-instantiated and assigns the one that results in the highest similarity.

\section{A. Search Space Analysis}

In order to comprehend the behavior of hill climbing under different search strategies, we first study the search space for configuration similarity. The goal is to identify how easily are local maxima found and how much they differ from random solutions. For the first experiment, we randomly selected five queries with 9 variables, and five with 12, and for each query 
we generated 500 random solutions in a dataset of 1,000 uniformly distributed rectangles with density 0.5 . Fig. 6 shows the average similarity of a solution and the average maximum and minimum similarities of the neighbors that can be reached with a single move. The similarity values are scaled, i.e., they are divided by the average maximum similarity found in each case. The $\mathrm{x}$-axis represents the five different queries, with no specific significance in the placement. According to the diagrams, there is a considerable difference between the similarity of a solution and the maximum and minimum similarity of its neighbors. For queries of size 9, this difference is around $15 \%$, while for queries involving 12 objects about $10 \%$. Thus, even a single move can have a significant effect on the quality of the solution especially in small queries (because each variable has a higher contribution to the total similarity).

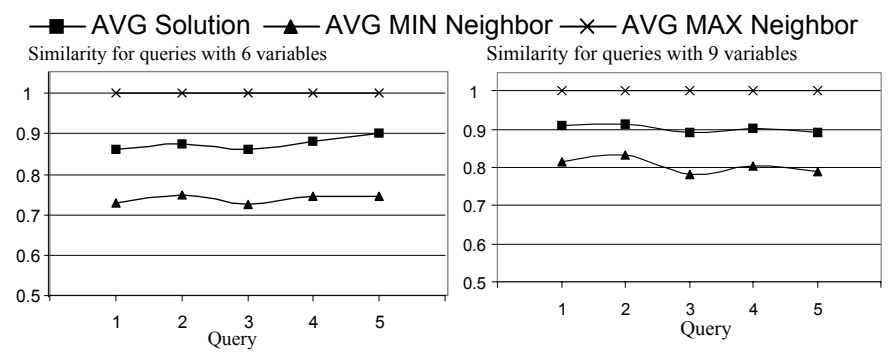

Fig. 6: Similarity ranges around random solutions

The second experiment studies the number of steps, i.e., uphill moves that must be performed in order to reach a local maximum. We use two approaches for identifying local maxima: (i) in the first one we replace a solution with the best of all its neighbors (ii) in the second one we accept the first better neighbor found by changing the instantiations of random variables. We refer to the local maxima obtained using these approaches as All-maximum (A-max for short) and First-maximum ( $F$-max) respectively. When searching for $A$ max, each step tries all possible values for each variable, i.e., a total of $\Theta(n \cdot N)$ attempts. For $F$-max this number differs in each step; in the best case an uphill move can be found with the first attempt, while in the worst, even $\mathrm{O}(n \cdot N)$ attempts may fail to find a better neighbor (some instantiations may be missed).

Fig. 7 shows how these maxima are reached (attempts, similarity) as a function of the number of steps, for a query with 9 variables over the 0.5 density dataset $\left(n=9, N=10^{3}\right)$. The horizontal axis corresponds to the number of steps, the left $y$-axis to the total number of attempts (including unsuccessful instantiations) and the right $y$-axis to similarity. $A$-max (similarity 0.824) is reached after 24 steps; 9,000 instantiations are tested in each step, resulting in 216,000 attempts. F-max (similarity 0.831 ) is reached after 77 steps and 273,408 attempts. Search for A-max is deterministic, meaning that starting with one seed, we always reach the same local maximum. On the other hand, the value of $F-\max$ and the steps required to reach it change depending on the order that neighbors are visited. In most cases the two maxima are close to each other.
Although search for F-max finds the highest similarity using a longer path (77 steps as opposed to 24), it reaches high quality solutions faster. Consider, for instance, a solution with similarity around 0.8 . If search is performed according to the A-max approach, the solution will be found after 9 steps and 81,000 attempts (see Fig. 7). On the other hand, if the F-max approach is employed, the solution will be found in about 40 steps. However, the total number of attempts is lower than 15,000 because uphill moves are easily performed from solutions of low similarity. The average number of attempts per step when searching for F-max is shown Fig. 8. Up to the $37^{\text {th }}$ step, fewer than 1,000 instantiations are needed before each uphill move is found ${ }^{4}$. At that point the similarity is close to 0.8 . Every attempt involves a similarity computation; thus the number of attempts (rather than steps) determines the cost of search. The advantage of the $F$-max search approach is clear, since it converges much faster to high similarity solutions.

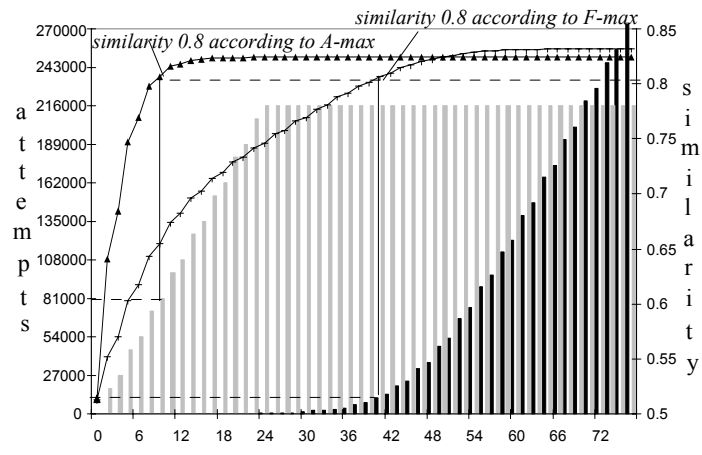

number of steps

\begin{tabular}{|c|c|c|}
\hline & $A-\max$ & $F-\max$ \\
\hline total attempts & & \\
\hline similarity & - & + \\
\hline
\end{tabular}

Fig. 7. Similarity and total number of attempts vs. the number of steps

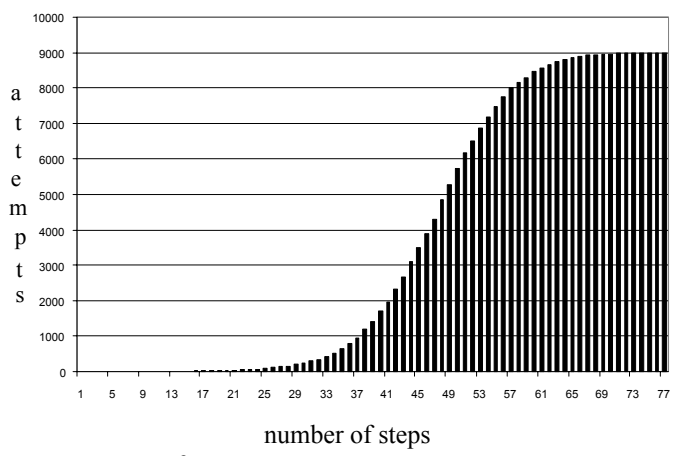

Fig. 8. Attempts per step for $F$-max

\section{B. Performance of Hill Climbing Alternatives}

According to the previous results, first-better value is expected to outperform all-best selection. However, as the quality of the solution increases, improvement by random reinstantiations becomes more difficult and large parts of the domains are searched (see Fig. 8). Near local maxima, firstbetter behaves likes all-best value selection, but unlike exhaustive search it may miss some good neighbors.

\footnotetext{
${ }^{4}$ The maximum number of attempts per step for this experiment is 9,000 , since there exist nine variables, each taking 1000 values.
} 

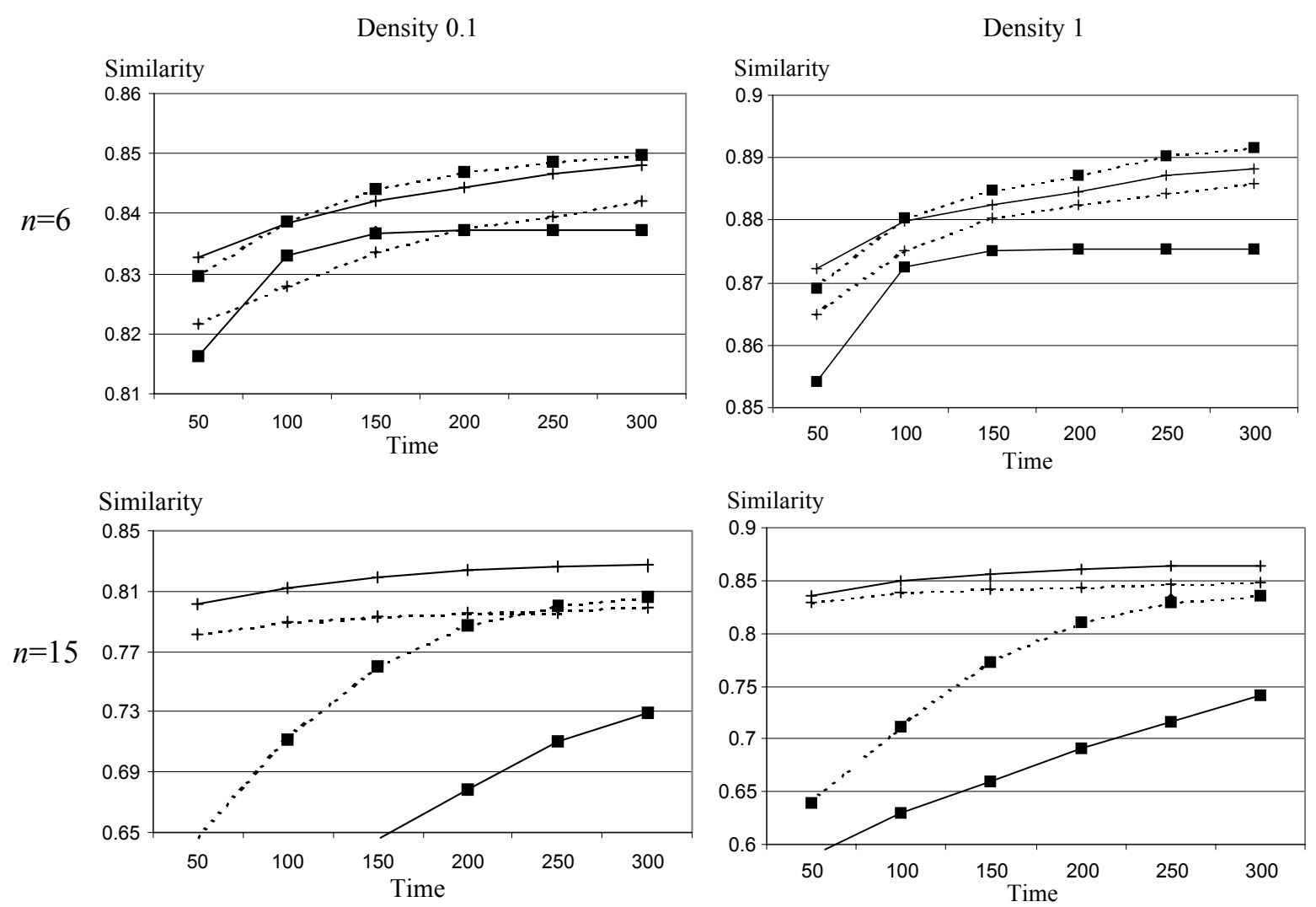

\begin{tabular}{|c|c|c|c|}
\hline variable & lection & $\begin{array}{l}\text { First-Better } \\
\text { F }\end{array}$ & $\begin{array}{c}\text { All-Best } \\
\text { A }\end{array}$ \\
\hline Random & $\mathbf{R}$ & + R-F & $\Rightarrow-\mathrm{R}-\mathrm{A}$ \\
\hline Worst & $\mathbf{W}$ & $\cdots+\cdots \mathrm{W}-\mathrm{F}$ & $\cdots \square \cdots \mathrm{W}-\mathrm{A}$ \\
\hline
\end{tabular}

Fig. 9. Similarity retrieved as a function of the execution time

Consequently, in some cases where there is enough time for processing (small queries and/or datasets), all-best may eventually yield better solutions than first-better selection.

In order to test this observation we ran experiments with the four variations of hill climbing ( 2 variable selection $\cdot 2$ value selection mechanisms) using the query sets of 6 and 15 variables over datasets of 1000 uniformly distributed rectangles with densities of 0.1 and $1^{5}$. Fig. 9 shows the average similarity (of 25 queries in each set) retrieved over the two datasets every 50 seconds.

Observe that the relative performance of the algorithms is the same for both datasets, despite the large density difference, implying that their effectiveness is independent of density. First-better value selection quickly (within the first 50 seconds) finds good solutions even for the large queries. Among the two variable selection mechanisms, random selection (R-F) is faster ${ }^{6}$ than worst variable (W-F) since random variables are more easily improved than the worst one. All-best value selection is ineffective for large queries because the number of neighbors, as well as the cost of

\footnotetext{
${ }^{5}$ Most real-life datasets have densities between 0.1 and 1 , with a density around 0.5 considered common/average. The datasets used in this section are synthetic (so we can vary the densities) and relatively small $\left(10^{3}\right.$ objects - so we can run numerous experiments). Nevertheless, similar results are obtained with the real datasets of section 4 .

${ }^{6} \mathrm{R}-\mathrm{F}$ corresponds to CSII algorithm.
}

similarity computations increases with the number of variables. Thus, $\mathrm{W}-\mathrm{A}$ and $\mathrm{R}-\mathrm{A}$ take a long time to converge to high similarity regions. Notice that for 15 variables W-A converges after 200 seconds, while R-A does not converge at all within the 300 -seconds limit. R-A is worse than W-A, because some variables, especially if the solution is good, contribute little, or not at all, to the total degree of inconsistency. Therefore, spending a long time to improve these variables does not pay-off.

For 6-variable queries, however, W-A (worst variable selection, all best value) outperforms CSII after 100 secs and achieves the highest similarity. This is due to a combination of reasons: for small queries, finding the best possible instantiation for a variable may increase the similarity of the solution significantly, especially if the variable chosen is the worst one. Furthermore, due to the small problem size, there is enough time to search extensively within the neighborhood of a solution, identifying good local maxima. Motivated by these observations, we propose an algorithm that can outperform the previous ones in all cases. The idea is to start with CSII that quickly reaches an area of high similarity. In subsequent steps (when CSII starts behaving like exhaustive search), a deterministic value selection technique locates the good neighbors, using the spatial structure of the problem to avoid the expensive search for all possible variable instantiations. 


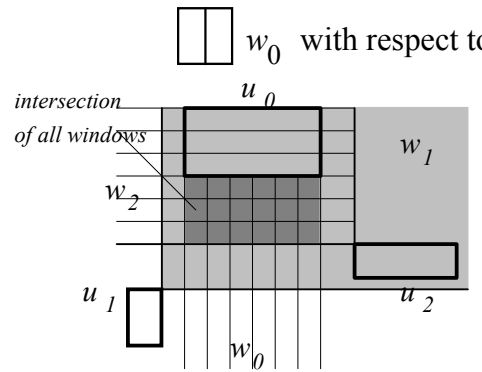

(a) exact windows

Fig. 10. Value selection using windows

\section{Window Value Selection}

Consider the example query of Fig. 1(a) where the first three variables are instantiated to objects $u_{0}, u_{1}$, and $u_{2}$, as shown in Fig. 10(a). Assume that these three instantiations perfectly match the query constraints. The fourth variable $\left(v_{3}\right)$ is chosen for re-instantiation and the goal is to find the best value for it. Variable $v_{3}$ is related with the other ones by the following projection-based constraints: $\operatorname{south}\left(v_{3}, v_{0}\right)$, northeast $\left(v_{3}, v_{l}\right)$ and northwest $\left(v_{3}, v_{2}\right)$. Each of the constraints, in combination with the current value of the corresponding variable, defines a window in space containing all consistent values for $v_{3}$ (e.g., all objects south of $v_{0}$ are inside $w_{0}$ ). The best values of $v_{3}$ (i.e., satisfying all constraints) are the ones that lie in the intersection of all windows. In other words, if a value is found in the dark gray area of Fig. 10(a), there is no need to search the whole domain of $v_{3}$.

Although, in the example of Fig. 10(a), we assume that the first three variables are instantiated to objects that result in a perfect match, in most cases the partial solution after the removal of a single variable, is only approximate. As an example consider the partial solution of Fig. 10(b), where $u_{0}$ has been shifted to the left. The instantiation $\left\{v_{0} \leftarrow u_{0}, v_{1} \leftarrow u_{1}, v_{2}\right.$ $\left.\leftarrow u_{2}\right\}$ has some inconsistency degree on the $x$ axis (the positions of the objects on the $y$ axis are the same). As a result, the intersection of $w_{0}, w_{1}$ and $w_{2}$ is empty and therefore cannot contain any objects. Intuitively, the good instantiations for $v_{3}$, are still found somewhere in the area between $u_{0}, u_{l}$, and $u_{2}$. In order to continue improving the solution, we should extend the windows so that a new value for $v_{3}$ can be chosen.

Window value selection (WVS) applies this idea. Once the variable $v_{i}$ for re-instantiation has been chosen, the appropriate windows $w_{j}(0 \leq j<n, i \neq j)$ are computed. Then each window is extended according to the maximum inconsistency degree $d$ of the partial solution (where all variables except for $v_{i}$ have been instantiated) on each dimension; the higher the value of $d$, the larger the extension on the corresponding axis. In the example of Fig. $10(\mathrm{c}), w_{0}, w_{1}$ and $w_{2}$ are only extended on the $x$-axis because there is no inconsistency on the $y$-axis. Although the objects in the intersection (dark gray area) do not result in perfect matches (e.g., the constraint between $v_{0}$ and $v_{l}$ is still violated), they provide good solutions that can be further improved in subsequent steps. The window extension method depends on the relation scheme in use. In the current implementation, which is based on conceptual neighbors [7],

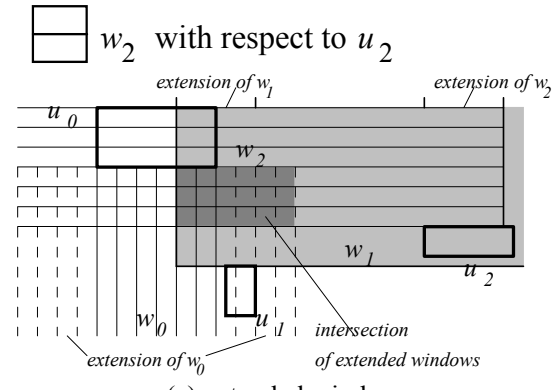

(c) extended windows

in addition to the original constraint, its $d$ neighbors are taken into account when generating the window. If angular directions were used, a northeast constraint, for instance, could generate an angular window $40^{\circ}-50^{\circ}$ in case of a low value of $d$, or a window $30^{\circ}-60^{\circ}$ for higher inconsistency.

In order to be able to search fast within such windows, all objects within an image are sorted according to the $x$ coordinate of the lower left point (this pre-processing takes place when the image is inserted in the database). The objects that fall inside the window (and potentially some false hits) are found by a simple range query in this sorted list. The other three co-ordinates of each retrieved object are checked and, if they also fall within the specified window, the object is kept as a good value. Initially, due to the low similarity of random solutions (seeds), the windows and their intersection usually cover the whole workspace. In this case WVS behaves like all-best value selection (with the additional overhead of computing the windows). As the inconsistency degree of the solution drops, the windows in some projections decrease restricting the search space.

We compare WVS against best-value selection (i.e., exhaustive domain search) for worst and random variable selection. Fig. 11 illustrates the results for query-sets (average of 25 queries per set) with 6 and 15 variables over the dataset with density $0.5 \quad\left(N=10^{3}\right)$. WVS always outperforms exhaustive search; as in the case of all-best value selection (see experiments in Fig. 9), WVS performs best with worst variable selection (W-WVS) since each re-instantiation may improve the solution significantly.

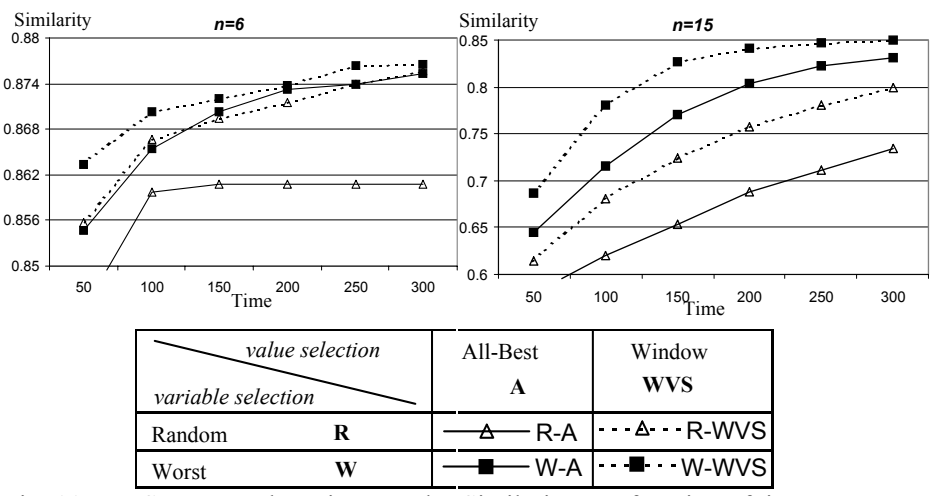

Fig. 11. WVS versus exhaustive search - Similarity as a function of time

\section{Two-Phase Search}

Despite its good performance, W-WVS does not converge 
fast to high similarity regions, due to the large windows in the initial phases of the algorithm. To circumvent this problem, we propose a two-phase search (2PS) algorithm that first uses CSII to quickly find a good solution, which is then improved by W-WVS. CSII is executed for a time analogous to the size of the problem (for this implementation $n \cdot N$ milliseconds), and $\mathrm{W}-\mathrm{WVS}$ for the remaining time. For instance, for a query with 15 variables over a 1000 objects dataset, the running time of CSII is 15 seconds. During this time CSII has performed enough steps to improve the seed significantly. Thus, in most cases the initial windows of W-WVS restrict the search to a relatively small portion of the space.

We compared 2PS with W-WVS and CSII using all query sets over the 0.5 density dataset $\left(N=10^{3}\right)$. As shown in Fig. 12, 2PS outperforms both algorithms in all cases since it combines their best characteristics. Observe that for small queries (6 and 9 variables), W-WVS produces better solutions than CSII even at the first 50 seconds. As the query size increases, WWVS slows down significantly and for 15 variables, it catches up with CSII only at 300 seconds.
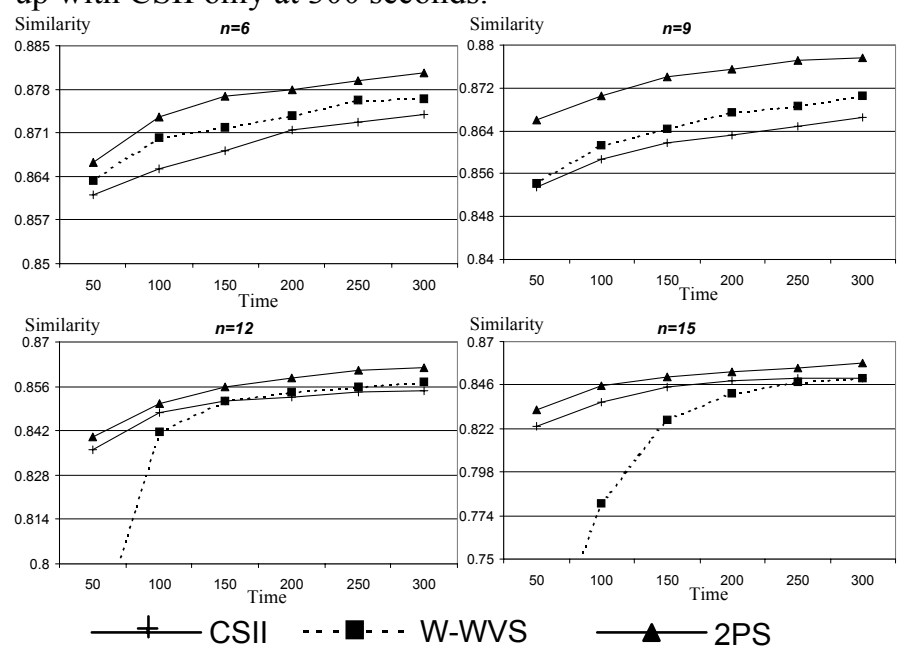

Fig. 12.2PS vs. WVS and CSII - Similarity as a function of time

TABLE I

SIMILARITY AS A FUNCTION OF TIME FOR RND AND FC

\begin{tabular}{|c|c|ccccccc|}
\hline \multirow{2}{*}{$\boldsymbol{n}$} & \multirow{2}{*}{ method } & \multicolumn{6}{|c|}{ Time (seconds) } \\
\cline { 3 - 9 } & & $\mathbf{5 0}$ & $\mathbf{1 0 0}$ & $\mathbf{1 5 0}$ & $\mathbf{2 0 0}$ & $\mathbf{2 5 0}$ & $\mathbf{3 0 0}$ \\
\hline \multirow{2}{*}{6} & RND & 0.742521 & 0.749104 & 0.752542 & 0.755187 & 0.756688 & 0.758604 \\
\cline { 2 - 8 } & FC & 0.6475 & 0.654167 & 0.691563 & 0.698229 & 0.703313 & 0.708438 \\
\hline \multirow{2}{*}{15} & RND & 0.665443 & 0.672321 & 0.678756 & 0.682307 & 0.683283 & 0.683923 \\
\cline { 2 - 8 } & FC & 0.566101 & 0.56692 & 0.56869 & 0.572113 & 0.575488 & 0.576732 \\
\hline
\end{tabular}

We also repeated the same experiment using RND and FC. Table 1 shows the best similarity retrieved over time for the smallest (6 variables) and largest (15 variables) query sizes. In general, both algorithms provide very low similarity when compared with the local search (see Fig. 12). RND produces better results than FC within the 300 seconds but quality does not increase much over time, implying that that only a small percentage of solutions have similarity close to a local maximum. Even though FC expectedly improves gradually with time it does not find good solutions even for 6 variables within the available limit. The situation is worse for 15 variables due to the significant increase of the search space; FC remains in the neighborhood of the initial assignments, which in most cases have low quality.

These results motivate the need for fast retrieval of suboptimal solutions in large multimedia databases. Among all techniques tested, 2PS consistently yielded the best performance for all combinations of queries/datasets (including real data). In addition to its robustness, another advantage of 2PS with respect to genetic algorithms and simulated annealing is that it does not require the complicated tuning of parameters that significantly affect efficiency.

\section{CONCLUSION}

This paper applies non-systematic search algorithms for processing configuration similarity queries. We first apply three techniques based on genetic algorithms, iterative improvement and simulated annealing, and compare them against forward checking, a very effective systematic search algorithm, and random search. Extensive experimentation, with various query/dataset combinations, shows that heuristic search is an effective way to process configuration similarity in cases that a near optimal solution is needed in restricted time. The best performance is consistently achieved by CSII, which is based on hill climbing; thus, as a second step we try to enhance its efficiency by studying alternative variable and value selection mechanisms. Next we present window value selection, a technique that quickly identifies the best values of a variable while avoiding exhaustive search in its domain. Finally, the best overall algorithm is shown to be two-phase search, a method that first applies CSII to quickly find a good solution and then improves it by window value selection.

To our knowledge, currently there do not exist other methods that can solve arbitrary queries (with no restrictions on the size of the problem or the type of the variables), given a time limit. The proposed methods have a wide range of applications in most modern spatial/multimedia database systems (which are increasingly vector-based), as well as the upcoming image/video compression methods such as MPEG4. An efficient way of indexing MPEG-4 video objects is proposed in [9]. In addition, some query languages such as Query-by-Sketch [8] and VisualSeek [34] already provide facilities for the expression of configuration similarity queries.

In our implementation we do not use any indexing for the input datasets. The application of a multi-dimensional data structure, such as R-trees, may improve the performance of heuristic search as it does for systematic algorithms [26]. In this way, the proposed algorithms are applicable in domains where the number of objects is very large (e.g., in the order of $10^{6}$ ). Future work can be carried out on the application of other sub-optimal algorithms (for a bibliography see [24]) on configuration similarity. In order to achieve efficiency, however, such algorithms need to be fine-tuned and modified for the particular, structure of the problem. 


\section{REFERENCES}

[1] J. Allen, "Maintaining knowledge about temporal intervals," $C A C M$, 26(11), pp. 832-843, 1983.

[2] S. Berchtold, C. Boehm, and H. Kriegel, "The pyramid technique: towards breaking the curse of dimensionality," in Proc. ACM SIGMOD, 1998, pp. 142-153,.

[3] S-K. Chang, , E. Jungert, and T. Li., "The design of pictorial databases based upon the theory of symbolic projections," in Proc. $1^{\text {st }}$ Symposium on Large Spatial Databases, 1989, pp. 303-323.

[4] S-K. Chang, Q. Shi, and C. Yan, "Iconic indexing by 2-D string," IEEE PAMI 9(3), pp. 413-428, 1987.

[5] V. Cerny, "Thermodynamical approach to the traveling salesman problem: an efficient simulation algorithm. J. Opt. Theory Appl., 45, pp. 41-51, 1985

[6] P. Ciaccia, M. Patella, and P. Zezula, "M-tree: an efficient access method for similarity search in metric spaces," in Proc. VLDB, 1997, pp. 426-435.

[7] V. Delis, D. Papadias, and N. Mamoulis, "Assessing multimedia similarity: a framework for structure and motion," in Proc. ACM Multimedia, 1998, pp. 333-338.

[8] M. Egenhofer, "Query processing in spatial-query-by-sketch," Journal of Visual Languages and Computing, Vol. 8, pp. 403-424, 1997.

[9] A. Ferman, B. Gunsel, and A. Tekalp, "Object-Based indexing of MPEG4 compressed video," in Proc. Visual Communications and Image Processing, SPIE, 1997, pp. 953-963.

[10] C. Freksa, "Temporal reasoning based on semi intervals," Artificial Intelligence, Vol 54, pp. 199-227, 1992

[11] C. Galindo-Legaria, A. Pellenkoft, and M. Kersten, "Fast, randomized join-order selection - why use transformations?," in Proc. VLDB, 1994, pp. 85-95.

[12] I. Gent, E. MacIntyre, P. Prosser, and T. Walsh, "The constrainedness of search." in Proc. AAAI, 1996, pp. 246-252.

[13] D. Goldberg, Genetic algorithms in search, optimization and machine learning. Addison-Wesley, Reading, Mass., 1989.

[14] J. Grefenstette, "Optimization of control parameters for genetic algorithms," IEEE Transactions on Systems, Man and Cybernetics, vol.16(1), pp. 122-128, 1986

[15] V. Gudivada, and V. Raghavan, "Design and evaluation of algorithms for image retrieval by spatial similarity." ACM Transactions on Information Systems, 13(1), pp. 115-144, 1995.

[16] R. Haralick, and G. Elliot, "Increasing tree search efficiency for constraint satisfaction problems," Artificial Intelligence, vol. 14, pp. 263-313, 1980

[17] Y. Ioannidis, and Y. Kang, "Randomized algorithms for optimizing large join queries," in Proc. ACM SIGMOD, 1990, pp. 312-321.

[18] S. Kirkpatrick, C. Gelat, and M. Vecchi, "Optimization by simulated annealing," Science, 220, pp. 671-680, 1983.

[19] S. Lee, and F. Hsu, "Spatial reasoning and similarity retrieval of images using 2D C-strings knowledge representation," Pattern Recognition, 25(3), pp. 305-318, 1992.

[20] S. Lee, M. Yang, and J. Chen, "Signature file as a spatial filter for iconic image database," Journal of Visual Languages and Computing, 3, pp. 373-397, 1992.

[21] S. Minton, M. Johnston, A. Philips, and P. Laird, "Minimizing conflicts: a heuristic method for constraint satisfaction and scheduling problems," Artificial Intelligence 58, pp. 161-205, 1992.

[22] M. Nabil, A. Ngu, and J. Shepherd, "Picture similarity retrieval using $2 \mathrm{~d}$ projection interval representation," IEEE TKDE, 8(4), pp. 533-539, 1996.

[23] S. Nahar, S. Sahni, and E. Shragowitz, "Simulated annealing and combinatorial optimization," in Proc. $23^{\text {rd }}$ Design Automation Conference, 1986, pp. 293-299.

[24] I. Osman, and G. Laporte, "Metaheuristics: a bibliography," Annals of Operations Research 63, pp. 513-623, 1996.

[25] D. Papadias, "Hill climbing algorithms for content-based retrieval of similar configurations," in Proc. ACM SIGIR, 2000, pp. 240-247.

[26] D. Papadias, N. Mamoulis, and V. Delis, "Algorithms for querying by spatial structure," in Proc. VLDB, 1998, pp. 546-557.

[27] D. Papadias, N. Mamoulis, and D. Meretakis, "Image similarity retrieval by spatial constraints," in Proc. ACM CIKM, 1998, pp. 289-296.
[28] D. Papadias, M. Mantzourogiannis, P. Kalnis, N. Mamoulis, and I. Ahmad, "Content-based retrieval using heuristic search," in Proc. ACM SIGIR, 1999, pp. 168-175.

[29] D. Papadias, and T. Sellis, "A pictorial query-by-example language," Journal of Visual Languages and Computing, Vol 6(1), pp. 53-72, 1995.

[30] E. Petrakis, and C. Faloutsos, "Similarity searching in medical image databases," IEEE TKDE, 9 (3), pp.435-447, 1997.

[31] E. Petrakis, C. Faloutsos, and K. Lin, "ImageMap: an image indexing method based on spatial similarity," IEEE TKDE, (to appear).

[32] K. Price, "Relaxation matching techniques - a comparison," IEEE PAMI 7, pp. 617-623, 1981.

[33] L. Shapiro, and R. Haralick, "Structural descriptions and inexact matching," IEEE PAMI 3, pp. 504-519, 1981.

[34] J. Smith, and S-F. Chang, "VisualSEEk: a fully automated content-based image query system," in Proc. ACM Multimedia, 1996, pp. 87-98.

[35] J. Smith, and S-F. Chang, "Integrated spatial and feature image query," Multimedia Systems 7, pp. 129-140, 1999.

[36] Srinivas, M., Patnaik, L. Adaptive Probabilities of Crossover and Mutation in Genetic Algorithms. IEEE Transactions on Systems, Man and Cybernetics, vol. 24(4), pp. 656-667, 1994

[37] A. Soffer, and H. Samet, "Retrieval by content in symbolic image databases," in Proc. Storage and Retrieval for Image and Video Databases, SPIE, 1996, pp. 144-155.

Dimitris Papadias is an assistant professor at the dept. of Computer Science, Hong Kong University of Science and Technology. Before joining HKUST in 1997, he studied and worked in several places including Queen's University (Canada), the National Technical University of Athens (Greece), the Technical University of Vienna (Austria), the University of California at San Diego, the National Center for Geographic Information and Analysis (USA), and the German National Research Center for Information Technology (GMD). His research interests include (spatial) databases, search algorithms and data warehousing.

Marios Mantzourogiannis obtained his B.Eng degree from the University of Patras, Greece and the M.Phil degree from the Hong Kong University of Science and Technology under the supervision of Dimitris Papadias and Ishfaq Ahmad. He is currently serving the Greek army.

Ishfaq Ahmad is an associate professor at the dept. of Computer Science, Hong Kong University of Science and Technology. He received his $\mathrm{PhD}$ degree in Computer Science from Syracuse University. His recent research focus has been on developing parallel programming tools, scheduling and mapping algorithms for scalable architectures, heterogeneous computing systems, distributed multimedia systems, video compression techniques, and web management. He is also the director of the Multimedia Technology Research Center, an officially recognized research center of HKUST, funded by various agencies of the Government of the Hong Kong Special Administrative Region as well as local and international industries. 